Kimberleigh B. Govender, Marivel Samipillai, Thavendran Govender, Hendrik G. Kruger and Tricia Naicker*

\title{
Crystal structure of 2-(bis(3,5-dimethylphenyl) ((methyldiphenylsilyl)oxy)methyl) pyrrolidine, $\mathrm{C}_{34} \mathrm{H}_{39} \mathrm{NOSi}$
}

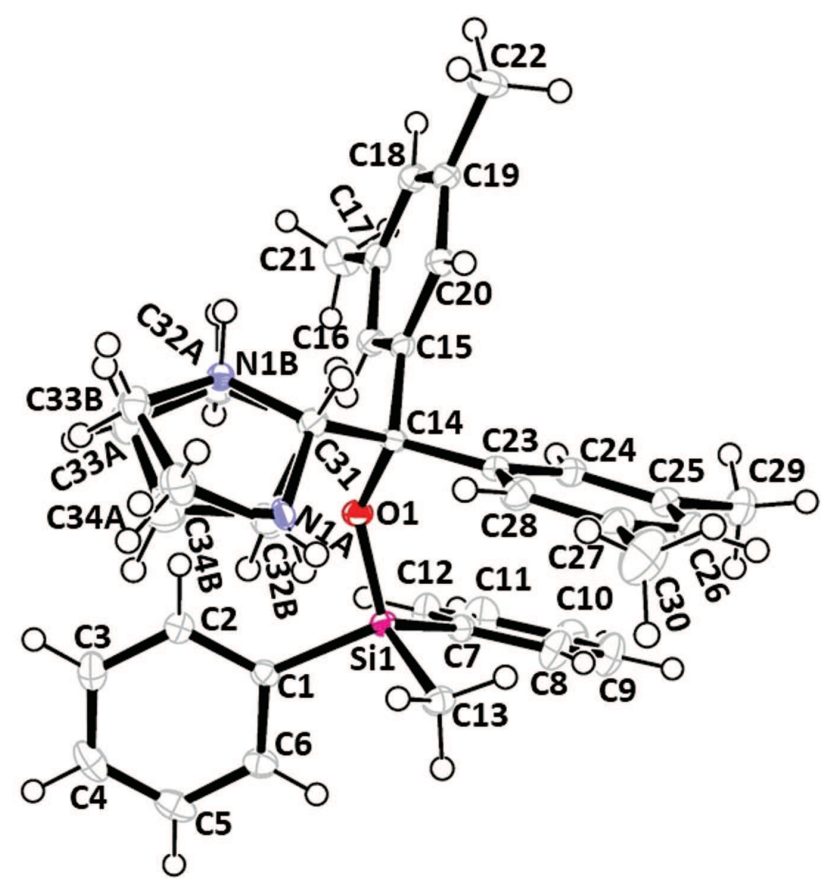

https://doi.org/10.1515/ncrs-2018-0422

Received October 11, 2018; accepted December 29, 2018; available online January 12, 2019

\section{Abstract \\ $\mathrm{C}_{34} \mathrm{H}_{39} \mathrm{NOSi}, \quad$ triclinic, $P \overline{1} \quad$ (no. 2), $\quad a=10.0901(2) \AA$, $b=11.5016(2) \AA, \quad c=14.4098(3) \AA, \quad \alpha=69.207(1)^{\circ}$, $\beta=86.555(2)^{\circ}, \quad \gamma=65.579(3)^{\circ}, \quad V=1416.10(6) \AA^{3}, \quad Z=2$, $R_{\mathrm{gt}}(F)=0.0463, w R_{\mathrm{ref}}\left(F^{2}\right)=0.1200, T=100(2) \mathrm{K}$.}

\section{CCDC no.: 1887917}

The crystal structure is shown in the figure. Tables 1 and 2 contain details on crystal structure and measurement conditions

*Corresponding author: Tricia Naicker, University of KwaZulu Natal, Catalysis and Peptide Research Unit, Durban 4000, South Africa, e-mail: naickert1@ukzn.ac.za

Kimberleigh B. Govender, Marivel Samipillai, Thavendran Govender and Hendrik G. Kruger: University of KwaZulu Natal, Catalysis and Peptide Research Unit, Durban 4000, South Africa
Table 1: Data collection and handling.
Crystal:

Size:

Wavelength:

$\mu$ :

Diffractometer, scan mode:

$\theta_{\max }$, completeness:

$N(h k l)_{\text {measured }}, N(h k l)_{\text {unique }}, R_{\text {int }}$ :

Criterion for $l_{\text {obs }}, N(h k l)_{\text {gt }}$ :

$N(\text { param })_{\text {refined }}$ :

Programs:

\author{
Colourless block \\ $0.37 \times 0.24 \times 0.13 \mathrm{~mm}$ \\ Mo $K \alpha$ radiation $(0.71073 \AA)$ \\ $0.11 \mathrm{~mm}^{-1}$ \\ Bruker APEX-II CCD, $\varphi$ and $\omega$ \\ $28.8^{\circ}, 99 \%$ \\ $39154,7216,0.020$ \\ $I_{\text {obs }}>2 \sigma\left(I_{\text {obs }}\right), 6613$ \\ 382 \\ COSMO [1], Bruker [2, 3], Olex2 \\ [4], ORTEP-3 [5], SHELX [6, 7]
}

and a list of the atoms including atomic coordinates and displacement parameters.

\section{Source of material}

A mixture of (R)- $\alpha, \alpha$-bis(3,5-dimethylphenyl)-2pyrrolidinemethanol (100 mg, 1.0 eq), DIEA (2.0 eq) and DMAP (0.2 eq) was stirred in anhydrous DCM $(1.6 \mathrm{~mL})$ at room temperature. Chloro(methyl)diphenylsilane (1.2 eq) was added and the reaction mixture was refluxed for $24 \mathrm{~h}$. The reaction was quenched with water and the resulting mixture was extracted twice with DCM. The combined organic layers were then washed once with brine, dried with $\mathrm{Na}_{2} \mathrm{SO}_{4}$ and concentrated. The crude material was then purified by column chromatography ( $20 \% \mathrm{EtOAc} / \mathrm{n}$-hexane, $\left.R_{\mathrm{f}}=0.2\right)$ to yield the title compound as an off-white solid (yield $56.2 \mathrm{mg}$, $34.4 \%)$. The product was dissolved in acetonitrile after the addition of heat and subsequent evaporation yielded colourless crystals suitable for X-ray analysis. m.p. $106-108{ }^{\circ} \mathrm{C}$. ${ }^{1} \mathrm{H}-\mathrm{NMR}\left(\mathrm{CDCl}_{3}, 400 \mathrm{MHz}\right): \delta[\mathrm{ppm}] 0.25$ (3H, s), $0.92-0.98$ $(1 \mathrm{H}, \mathrm{m}), 1.17-1.21(1 \mathrm{H}, \mathrm{m}), 1.47-1.53(2 \mathrm{H}, \mathrm{m}), 1.66-1.73(1 \mathrm{H}$, $\mathrm{m}), 2.25(12 \mathrm{H}, \mathrm{s}), 2.60-2.65(1 \mathrm{H}, \mathrm{m}), 2.77-2.81(1 \mathrm{H}, \mathrm{m}), 3.99$ $-4.02(1 \mathrm{H}, \mathrm{t}, \mathrm{J}=4.52 \mathrm{~Hz}), 6.86-6.89(2 \mathrm{H}, \mathrm{d}, \mathrm{J}=11.8 \mathrm{~Hz}), 6.99$ $(2 \mathrm{H}, \mathrm{s}), 7.10(2 \mathrm{H}, \mathrm{s}), 7.32-7.37(6 \mathrm{H}, \mathrm{m}), 7.54(4 \mathrm{H}, \mathrm{s})$.

\section{Experimental details}

All hydrogen atoms attached to carbon were placed in idealised positions and refined using the riding model with 
Table 2: Fractional atomic coordinates and isotropic or equivalent isotropic displacement parameters $\left(\AA^{2}\right)$.

\begin{tabular}{|c|c|c|c|c|}
\hline Atom & $x$ & $y$ & $z$ & $\boldsymbol{U}_{\text {iso }}{ }^{*} / \boldsymbol{U}_{\mathrm{eq}}$ \\
\hline ji1 & $0.24984(4)$ & $0.26137(4)$ & $0.31410(3)$ & $0.01343(9)$ \\
\hline 01 & $0.16889(10)$ & $0.24233(10)$ & $0.22865(7)$ & $0.01643(19)$ \\
\hline 1 & $0.24404(14)$ & $0.43638(14)$ & $0.25001(10)$ & $0.0156(2)$ \\
\hline C2 & $0.20539(16)$ & $0.50797(15)$ & $0.14742(11)$ & $0.0200(3)$ \\
\hline 2 & 0.169589 & 0.472150 & 0.109640 & $0.024^{\star}$ \\
\hline $\mathrm{C} 3$ & $0.21842(17)$ & $0.63098(16)$ & $0.09954(12)$ & $0.0252(3)$ \\
\hline 3 & 0.192336 & 0.677778 & 0.029678 & $0.030^{*}$ \\
\hline 24 & $0.26938(17)$ & $0.68493(15)$ & $0.15382(13)$ & $0.0270(3)$ \\
\hline 4 & 0.280405 & 0.767617 & 0.120979 & $0.032^{\star}$ \\
\hline $\mathrm{C} 5$ & $0.30431(17)$ & (16) & 0.256 & $0.0260(3)$ \\
\hline $\mathrm{H} 5$ & 0.336412 & 0.656353 & 0.293926 & $0.031 *$ \\
\hline C6 & 0.292 & $0.49487(15)$ & 0.303 & $0.0204(3)$ \\
\hline H6 & 0.317045 & 0.449353 & 0.373473 & $0.024^{*}$ \\
\hline $\mathrm{C} 7$ & (15) & (14) & (10) & $0.0166(3)$ \\
\hline $\mathrm{C} 8$ & $0.20076(17)$ & $0.14263(16)$ & $0.51584(11)$ & $0.0250(3)$ \\
\hline $\mathrm{H} 8$ & 0.287169 & 0.065095 & 0.517211 & $0.030^{\star}$ \\
\hline C9 & 0.12885 & $0.13837(18)$ & 0.6015 & $06(4)$ \\
\hline 19 & 0.165571 & .058342 & 0.660209 & $.037^{\star}$ \\
\hline 10 & $0.00405(18)$ & $0.25057(18)$ & 0.60121 & $0.0276(3)$ \\
\hline 10 & -0.045216 & 0.248382 & 0.659677 & $.033^{*}$ \\
\hline 11 & $-0.04872(18)$ & $0.36595(18)$ & $0.51539(13)$ & $98(3)$ \\
\hline 11 & -0.134482 & 0.443449 & 0.514978 & $0.036^{\star}$ \\
\hline 12 & 0.022 & 0.369 & 0.42 & $0.0240(3)$ \\
\hline 1 & -0.0 & 692 & 823 & $.029^{\star}$ \\
\hline C13 & 0.444 & 0.13 & 0.3 & 89(3) \\
\hline $13 A$ & 0.44 & 0.044480 & 504 & $0.028^{*}$ \\
\hline H13E & 0.488801 & 920 & 6733 & $.028^{*}$ \\
\hline $\mathrm{H} 13 \mathrm{C}$ & 0.49 & 07 & 187 & $.028^{\star}$ \\
\hline C14 & $0.17844(14)$ & & 0.19 & 40(2) \\
\hline 15 & 0.0 & & (9) & 42(2) \\
\hline C16 & -0.099 & 0.256 & 0.19 & $.0167(3)$ \\
\hline H16 & -0.08 & 58 & 723 & $0.020^{*}$ \\
\hline 17 & -0.2406 & 0.2690 & (10) & 91(3) \\
\hline C18 & $-0.25634(15)$ & $0.16895(15)$ & $0.14886(10)$ & $0.0201(3)$ \\
\hline 18 & -0.351798 & & 799 & $0.024^{*}$ \\
\hline C19 & $-0.13552(16)$ & $0.05747(15)$ & $0.14103(10)$ & $.0189(3)$ \\
\hline & 0.0038 & & 0.1591 & $0.0168(3)$ \\
\hline $\mathrm{H} 2 \mathrm{O}$ & 0.087310 & -0.028974 & 0.154524 & $0.020^{*}$ \\
\hline C21 & $-0.37319(17)$ & $0.39093(17)$ & $0.17909(14)$ & $0.0295(3)$ \\
\hline $\mathrm{H} 21 \mathrm{~A}$ & -0.342892 & 0.441812 & 0.208782 & $0.044^{*}$ \\
\hline $\mathrm{H} 21 \mathrm{E}$ & -0.441381 & 0.359419 & 220190 & $0.044^{\star}$ \\
\hline $\mathrm{H} 21 \mathrm{C}$ & -0.421480 & 0.450817 & 0.111651 & $0.044^{\star}$ \\
\hline $\mathrm{C} 22$ & $-0.15428(19)$ & $-0.05095(18)$ & $0.11456(13)$ & $0.0285(3)$ \\
\hline $\mathrm{H} 22 \mathrm{~A}$ & -0.257165 & -0.019008 & 0.091780 & $0.043^{*}$ \\
\hline $\mathrm{H} 22 \mathrm{~B}$ & -0.124437 & -0.135568 & 0.173476 & $0.043^{*}$ \\
\hline $\mathrm{H} 22 \mathrm{C}$ & -0.093407 & -0.068681 & 0.061224 & $0.043^{*}$ \\
\hline C23 & $0.26844(15)$ & $-0.00592(14)$ & $0.28037(10)$ & $0.0157(3)$ \\
\hline C24 & $0.21040(15)$ & $-0.03995(15)$ & $0.37124(10)$ & $0.0188(3)$ \\
\hline H24 & 0.115898 & 0.020886 & 0.379018 & $0.023^{\star}$ \\
\hline $\mathrm{C} 25$ & $0.28773(17)$ & $-0.16105(16)$ & $0.45087(11)$ & $0.0224(3)$ \\
\hline C26 & $0.42515(18)$ & $-0.24967(15)$ & $0.43735(12)$ & $0.0248(3)$ \\
\hline H26 & 0.479157 & -0.332604 & 0.491052 & $0.030^{*}$ \\
\hline $\mathrm{C} 27$ & $0.48530(17)$ & $-0.21987(16)$ & $0.34728(12)$ & $0.0246(3)$ \\
\hline $\mathrm{C} 28$ & $0.40518(16)$ & $-0.09761(14)$ & $0.26884(11)$ & $0.0200(3)$ \\
\hline $\mathrm{H} 28$ & 0.444621 & -0.076685 & 0.206654 & $0.024^{\star}$ \\
\hline
\end{tabular}

Table 2 (continued)

\begin{tabular}{|c|c|c|c|c|}
\hline Atom & $x$ & $y$ & $z$ & $U_{\text {iso }} * / U_{\text {eq }}$ \\
\hline C29 & $0.2244(2)$ & $-0.19454(19)$ & $0.54926(12)$ & $0.0313(4)$ \\
\hline $\mathrm{H} 29 \mathrm{~A}$ & 0.244719 & -0.149263 & 0.589214 & $0.047^{\star}$ \\
\hline H29B & 0.268831 & -0.293935 & 0.585315 & $0.047^{\star}$ \\
\hline $\mathrm{H} 29 \mathrm{C}$ & 0.118332 & -0.162176 & 0.537182 & $0.047^{*}$ \\
\hline C30 & $0.6348(2)$ & $-0.31696(19)$ & $0.33413(15)$ & $0.0400(4)$ \\
\hline $\mathrm{H} 30 \mathrm{~A}$ & 0.710207 & -0.308312 & 0.367539 & $0.060^{\star}$ \\
\hline $\mathrm{H} 30 \mathrm{~B}$ & 0.647374 & -0.294286 & 0.262855 & 0.060 * \\
\hline $\mathrm{H} 30 \mathrm{C}$ & 0.643965 & -0.411275 & 0.363473 & $0.060^{*}$ \\
\hline C31 & $0.23985(14)$ & $0.15513(14)$ & $0.09626(10)$ & $0.0171(3)$ \\
\hline $\mathrm{H} 31^{\mathrm{a}}$ & 0.248681 & 0.078992 & 0.074505 & $0.020^{*}$ \\
\hline$H 31 A^{b}$ & 0.264682 & 0.071567 & 0.079691 & $0.020^{*}$ \\
\hline $\mathrm{N} 1 \mathrm{~A}^{\mathrm{a}}$ & $0.3830(5)$ & $0.1621(5)$ & $0.0937(3)$ & $0.0159(8)$ \\
\hline $\mathrm{H} 1 \mathrm{~A}^{\mathrm{c}}$ & $0.405(3)$ & $0.1771(17)$ & $0.1460(9)$ & $0.019^{*}$ \\
\hline$C 32 A^{a}$ & $0.1337(8)$ & $0.2918(7)$ & $0.0192(5)$ & $0.0245(16)$ \\
\hline $\mathrm{H} 32 \mathrm{~A}^{\mathrm{a}}$ & 0.060440 & 0.277980 & -0.013147 & $0.029^{*}$ \\
\hline $\mathrm{H} 32 \mathrm{~B}^{\mathrm{a}}$ & 0.081621 & 0.358603 & 0.051815 & 0.029 * \\
\hline$C 33 A^{a}$ & $0.2251(6)$ & $0.3433(6)$ & $-0.0569(4)$ & $0.0305(12)$ \\
\hline $\mathrm{H} 33 \mathrm{~A}^{\mathrm{a}}$ & 0.225359 & 0.316806 & -0.115195 & $0.037^{\star}$ \\
\hline$H 33 B^{a}$ & 0.185912 & 0.444432 & -0.080130 & $0.037^{\star}$ \\
\hline$C 34 A^{d}$ & $0.3696(8)$ & $0.2812(8)$ & $-0.0077(5)$ & $0.0397(16)$ \\
\hline$H 34 A^{d}$ & 0.391111 & 0.350831 & 0.005408 & $0.048^{*}$ \\
\hline$H 34 B^{d}$ & 0.441600 & 0.244181 & -0.051162 & $0.048^{*}$ \\
\hline $\mathrm{N} 1 \mathrm{~B}^{\mathrm{b}}$ & $0.1379(7)$ & $0.2769(6)$ & $0.0135(4)$ & $0.0133(13)$ \\
\hline $\mathrm{H} 1 \mathrm{~B}^{\mathrm{c}}$ & $0.070(2)$ & $0.262(3)$ & $-0.011(2)$ & $0.016^{*}$ \\
\hline $\mathrm{C} 32 \mathrm{~B}^{\mathrm{b}}$ & $0.3764(10)$ & $0.1884(5)$ & $0.0866(6)$ & $0.0226(12)$ \\
\hline $\mathrm{H} 32 \mathrm{C}^{\mathrm{b}}$ & 0.360886 & 0.262434 & 0.111669 & $0.027^{\star}$ \\
\hline$H 32 D^{b}$ & 0.465234 & 0.106269 & 0.123712 & $0.027^{*}$ \\
\hline $\mathrm{C} 33 \mathrm{~B}^{\mathrm{b}}$ & $0.2300(6)$ & $0.3191(6)$ & $-0.0646(4)$ & $0.0222(12)$ \\
\hline $\mathrm{H} 33 \mathrm{C}^{\mathrm{b}}$ & 0.206200 & 0.417671 & -0.083373 & $0.027^{*}$ \\
\hline$H 33 D^{b}$ & 0.214111 & 0.302540 & -0.125005 & $0.027^{\star}$ \\
\hline $\mathrm{C} 34 \mathrm{~B}^{\mathrm{e}}$ & $0.3878(3)$ & $0.2312(3)$ & $-0.01772(18)$ & $0.0254(5)$ \\
\hline $\mathrm{H} 34 \mathrm{C}^{\mathrm{e}}$ & 0.433403 & 0.151320 & -0.038799 & $0.030^{*}$ \\
\hline$H 34 D^{e}$ & 0.446683 & 0.285265 & -0.036745 & 0.030 * \\
\hline
\end{tabular}

a Occupancy: 0.556(12), ${ }^{\mathrm{b}}$ Occupancy: 0.444(12), ' Occupancy: 0.5,

${ }^{\mathrm{d} O}$ Occupancy: 0.3, ${ }^{\mathrm{e}}$ Occupancy: 0.7 .

$U_{\text {iso }}$ values set to 1.2 or 1.5 times of those of their parent atoms and $\mathrm{C}-\mathrm{H}$ distances constrained ranging from $0.95 \AA$ to $1.00 \AA$. Hydrogen atoms attached to nitrogen were added manually and their positions refined subject to restraints.

\section{Comment}

Diarylprolinol silyl ethers have become synonymous with organocatalysis. Developed independently by Jørgensen [8] and Hayashi [9], diarylprolinol silyl ethers have shown to be successful as both enamine and iminium activators [10]. They have been applied to a vast array of reactions [11-13] and have led to the development of many derivatives [14-16]. Our group has shown interest in the field of organocatalysis and its applications [17] and we are now developing novel 
diarylprolinol silyl ethers to be incorporated in asymmetric organocatalytic reactions. We have here described the crystal structure of the title compound.

The crystal structure of the title compound contains one molecule in the asymmetric unit and the pyrrolidine moiety is disordered over two positions. The phenyl rings attached to the Si-atom of the title compound are slightly twisted with the torsion angles of $104.66(13)^{\circ}$ for C8-C7Si1-01 and $-11.66(13)^{\circ}$ for C2-C1-Si1-01. Similarly the two dimethylphenyl moieties attached to the quaternary carbon (C14) of the molecule and are also slightly twisted with the torsion angles of $-112.05(14)^{\circ}$ for $\mathrm{C} 28-\mathrm{C} 23-\mathrm{C} 14-\mathrm{O} 1$ and $4.99(17)^{\circ}$ for $\mathrm{C} 16-\mathrm{C} 15-\mathrm{C} 14-\mathrm{O} 1 . \mathrm{C}-\mathrm{H} \cdots \pi$ interactions are found to play a crucial role to interconnect the adjacent molecules in the crystal lattice.

\section{References}

1. COSMO, V1.61. Bruker AXS Inc., Madison, WI (2009).

2. Bruker. SAINT-Plus, SADABS. Bruker AXS Inc., Madison, WI, USA (2008).

3. Bruker. APEX2, XPREP. Bruker AXS Inc., Madison, WI, USA (2008).

4. Dolomanov, O. V.; Bourhis, L. J.; Gildea, R. J.; Howard, J. A. K.; Puschmann, H.: OLEX2: a complete structure solution, refinement and analysis program. J. Appl. Crystallogr. 42 (2009) 339-341.

5. ORTEP-3 for Windows with a Graphical User Interface (GUI). J. Appl. Crystallogr. 45 (2012) 849-854.

6. Sheldrick, G. M.: Crystal structure refinement with SHELXL. Acta Crystallogr. C71 (2015) 3-8.
7. Sheldrick, G. M.: A short history of SHELX. Acta Crystallogr. A64 (2008) 112-122.

8. Marigo, M.; Wabnitz, T. C.; Fielenbach, D.; Jørgensen, K. A.: Enantioselective organocatalyzed $\alpha$-sulfenylation of aldehydes. Angew. Chem. Int. Ed. 44 (2005) 794-797.

9. Hayashi, Y.; Gotoh, H.; Hayashi, T.; Shoji, M.: Diphenylprolinol silyl ethers as efficient organocatalysts for the asymmetric Michael reaction of aldehydes and nitroalkenes. Angew. Chem. Int. Ed. 44 (2005) 4212-4215.

10. Bertelsen, S.; Jørgensen, K. A.: Organocatalysis - after the gold rush. Chem. Soc. Rev. 38 (2009) 2178-2189.

11. Jensen, K. L.; Dickmeiss, G.; Jiang, H.; Albrecht, L.; Jørgensen, K. A.: The diaryl prolinol silyl ether system: a general organocatalyst. Acc. Chem. Res. 45 (2012) 248-264.

12. Klier, L.; Tur, F.; Poulsen, P. H.; Jørgensen, K. A.: Asymmetric cycloaddition reactions catalysed by diarylprolinol silyl ethers. Chem. Soc. Rev. 46 (2017) 1080-1102.

13. Xu, L.-W.; Li, L.; Shi, Z.-H.: Asymmetric synthesis with siliconbased bulky amino organocatalysts. Adv. Synth. Catal. 352 (2010) 243-279.

14. Donslund, B. S.; Johansen, T. K.; Poulsen, P. H.; Halskov, K. S.; Jørgensen, K. A.: The diarylprolinol silyl ethers: ten years after. Angew. Chem. Int. Ed. 54 (2015) 13860-13874.

15. Lombardo, M.; Montroni, E.; Quintavalla, A.; Trombini, C.: A new family of conformationally constrained bicyclic diarylprolinol dilyl ethers as organocatalysts. Adv. Synth. Catal. 354 (2012) 3428-3434.

16. Marques-Lopez, P. H.; Raquel, E.: Diarylprolinol derivatives in organocatalysis from another point of view: structural aspects. Curr. Org. Chem. 15 (2011) 2311-2327.

17. Cele, Z. E. D.; Arvidsson, P. I.; Kruger, H. G.; Govender, T.; Naicker, T.: Applied enatioselective aminocatalysis: $\alpha$ heteroatom functionalization reactions on the carbapenem ( $\beta$-lactam antibiotic) core. Eur. J. Org. Chem. 2015 (2015) 638-646. 